\title{
Characterization of Inorganic Silicalite-1 Membrane to be used for the Separation of Greenhouse Gases
}

\author{
David Carter, Dean Kennedy, F. Handan Tezel, Boguslaw Kruczek \\ University of Ottawa, Department of Chemical and Biological Engineering \\ 161 Louis Pasteur, Ottawa, Ontario, Canada K1N 6N5 \\ dcart028@uottawa.ca,dkenn022@uottawa.ca, handan.tezel@uottawa.ca,bkruczek@uottawa.ca
}

\begin{abstract}
Defect free MFI type silicalite-1 membranes have been fabricated inside tubular $\mathrm{TiO}_{2}$ ceramic supports to be used for the separation of greenhouse gases. In this work, single gas permeance experiments have been conducted. The results of which in conjunction with adsorption isotherms for each gas investigated have been used to calculate the effective diffusivities of $\mathrm{N}_{2}, \mathrm{CH}_{4}$, and $\mathrm{CO}_{2}$. The permeances at $22{ }^{\circ} \mathrm{C}$ and 1 atm of pressure differential for $\mathrm{He}, \mathrm{N}_{2}, \mathrm{CO}_{2}$ and $\mathrm{CH}_{4}$, were found to be $4.38 \times 10^{-7}, 7.71 \times 10^{-7}, 1.35 \times 10^{-6}$, and $1.64 \times 10^{-6}$ $\mathrm{mol} / \mathrm{m}^{2} \mathrm{sPa}$, respectively, giving permeance ratios for the separation of $\mathrm{CH}_{4} / \mathrm{N}_{2}, \mathrm{CO}_{2} / \mathrm{N}_{2}$, and $\mathrm{CH}_{4} / \mathrm{CO}_{2}$ equal to $2.13,1.75$, and 1.22 respectively. These permeance ratios are comparable to those found in other studies reported in the literature, whereas the permeances are typically 1 to 2 orders of magnitude greater than those found in the literature at similar conditions.
\end{abstract}

Keywords: Inorganic membranes, Silicalite, characterization, Diffusivity, Surface diffusion, Adsorption, Carbon dioxide, Methane, Nitrogen.

(C) Copyright 2014 Authors - This is an Open Access article published under the Creative Commons Attribution License terms (http://creativecommons.org/licenses/by/3.0). Unrestricted use, distribution, and reproduction in any medium are permitted, provided the original work is properly cited.

\section{Introduction}

In order to mitigate the effects of global warming, a variety of carbon capture technologies are required which are economically favourable [1]. Inorganic membranes and MFI type silicalite-1 membranes in particular can tolerate extremely acidic conditions, and can withstand high pressures which make them suitable for the separation of $\mathrm{CO}_{2}$ and $\mathrm{CH}_{4}$ from gaseous process streams [2].

The inorganic membranes used in this study are fabricated by impregnating silicalite- 1 crystals into the pores of $\mathrm{TiO}_{2}$ membrane supports up to a depth of approximately $5 \mu \mathrm{m}$ to create a thin selective layer. By minimizing the thickness of selective layer, a membrane is produced which has a high permeance, and is able to process large volumes. There exists a trade-off between permeance and permselectivity, the preferential interaction of one component with the membrane. The trade-off can be modelled and used to design membranes for specific processes by characterizing various inorganic membranes [3]. To this end, an understanding of the transport of gas molecules through MFI type silicalite- 1 membranes is required, which is investigated in this study.

\section{Theory}

The silicalite- 1 crystals synthesized in this study have zeolite type structure. That is, they contain a uniformly distributed network of pores that in this case have a single pore size with no pore size distribution. This 3D lattice structure results in a large internal surface area, with an internal pore size of $5.5 \AA$. This pore size is less than twice the kinetic diameter of the gases investigated in this study: $3.3 \AA$, $3.6 \AA$, and $3.8 \AA$ for $\mathrm{CO}_{2}, \mathrm{~N}_{2}$, and $\mathrm{CH}_{4}$, respectively, and so it is assumed in this study that monolayer, and not multilayer adsorption is occurring, and that the main mechanism of gas transport at room temperature is surface diffusion. By this mechanism, gas molecules are adsorbed inside the pores of the silicalite- 1 crystals, and move in the direction of decreasing surface occupancy.

For monolayer adsorption of $\mathrm{N}_{2}, \mathrm{CH}_{4}$, and $\mathrm{CO}_{2}$ into active sites inside a single pore of silicalite-1, a randomly oriented monolayer, similar to a single file system is generated, and in this case, diffusion can be described by Fick's laws [4]. A version of Fick's law has 
therefore been used to calculate the effective diffusivity using the following equation for defect free membranes.

$J=D_{e}\left(q_{f}-q_{p}\right) A$

In this equation, $J$ represents the membrane flux $\left[\mathrm{mol} / \mathrm{m}^{2} / \mathrm{s}\right], D_{e}$ is the effective diffusivity $\left[\mathrm{m}^{2} / \mathrm{s}\right]$, and $A$ is the constant parameter for a single membrane which takes into account the physical characteristics of the membrane including membrane thickness and tortuosity. $q_{f}-q_{p}$ represents the driving force, which has been calculated as the gradient of surface occupancy, or working capacity of silicalite- 1 from the feed side membrane pressure to the permeate side membrane pressure. Given the dependence of $q_{f}$ and $q_{p}$ on temperature, volumetrically determined adsorption capacities found by Li \& Tezel [5], [6] were modified using the Temperature Dependant Sips model as described by Ahmadpour et al. [7] for the estimation of the adsorption capacities at room temperature. The general equation for the Temperature Dependant Sips model is as follows:

$$
q=q_{s} \frac{(b p)^{\frac{1}{n}}}{1+(b p)^{\frac{1}{n}}}
$$

In this equation, $p$ represents pressure [atm], $q_{s}$ is the adsorption capacity at saturation [mmol/g of adsorbent], $q$ is the adsorption capacity at a pressure $p$ [mmol/g of adsorbent], and $b$ and $n$ are constants which have units of [atm ${ }^{-1}$ ] and [dimensionless], respectively.

The pressure gradient normalized flux is referred to as permeance, and has units of $\left[\mathrm{mol} / \mathrm{m}^{2} \mathrm{sPa}\right]$.

\section{Experimental}

\subsection{Membrane Fabrication}

The interrupted pore plugging method was used to synthesise defect free MFI type silicalite-1 membranes on $\mathrm{TiO}_{2}$ ceramic membrane supports as reported by Miachon et al. [8]. Some modifications to the reported procedure were made, and are as follows:

- Following centrifugation of the precursor solution, supernatant liquid is added to the Teflon beaker, and topped up after a period of 3 hours instead of 10 minutes in order to ensure that the membranes' supports have absorbed as much precursor solution as possible at room temperature and pressure.
- For hydrothermal synthesis, the oven was not preheated, and was instead heated up to $170{ }^{\circ} \mathrm{C}$ at a rate of $1{ }^{\circ} \mathrm{C} / \mathrm{min}$ with the autoclave module inside the oven from the start.

- The reported standard calcination procedure was used with a heating up rate of $1{ }^{\circ} \mathrm{C} / \mathrm{min}$ instead of $1.7^{\circ} \mathrm{C} / \mathrm{min}$.

\subsection{Single Gas Permeation Experiments}

Before commencing each single gas permeation experiment, the membrane was purged with $\mathrm{He}$ at a flow rate of $100 \mathrm{cc}(\mathrm{STP}) / \mathrm{min}$ and a feed side pressure of $2 \mathrm{~atm}$ for 16 hours. Each gas was then investigated in turn for successively greater feed pressures between 1 atm and 5 atm. The gases of interest were tested in order of increasing adsorption capacity on silicalite- 1 as found by Li \& Tezel [5], [6], which is $\mathrm{N}_{2}, \mathrm{CH}_{4}$, and $\mathrm{CO}_{2}$.

\section{Results and Discussion}

\subsection{Adsorption Isotherms for $\mathrm{N}_{2}, \mathrm{CH}_{4}$, and $\mathrm{CO}_{2}$}

As described in the previous section, adsorption isotherms were generated using the Temperature Dependant Sips model which is based on Equation 2 for $\mathrm{N}_{2}, \mathrm{CH}_{4}$, and $\mathrm{CO}_{2}$ on silicalite- 1 at a temperature of $\mathrm{T}=$ $22^{\circ} \mathrm{C}$.

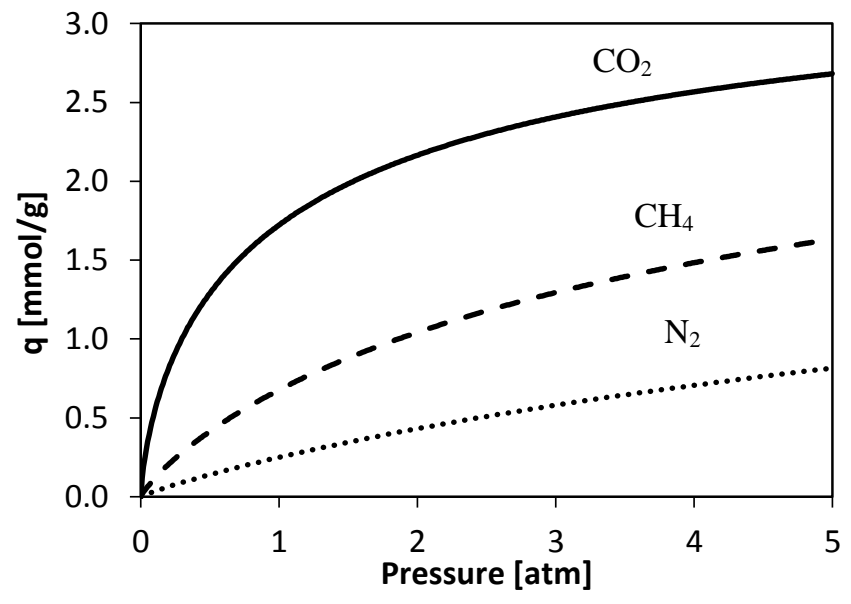

Figure 1. Adsorption isotherms for gases on silicalite- 1 at $\mathrm{T}=$ $22{ }^{\circ} \mathrm{C}$ generated using the Temperature Dependant Sips model and the data from Li \& Tezel [5], [6] as described by Ahmadpour et al. [7].

Figure 1 shows the expected order of increasing adsorption capacity $\mathrm{q}$ for the gases studied in this paper. He gas is assumed to be not adsorbed in silicalite-1. The reported isotherms from which these isotherm curves were generated are for particles and not membranes, however, the constant $\mathrm{A}$ in Equation 2 is assumed to account for this variation. 


\subsection{Single Gas Permeances for $\mathrm{He}, \mathrm{N}_{2}, \mathrm{CH}_{4}$, and $\mathrm{CO}_{2}$}

The single gas permeances for $\mathrm{He}, \mathrm{N}_{2}, \mathrm{CH}_{4}$, and $\mathrm{CO}_{2}$ were found for a silicalite- 1 membrane on a $0.8 \mu \mathrm{m}$ $\mathrm{TiO}_{2}$ support and are shown in Figure 2.

Defects are confirmed to be absent from the membrane by analysing the permeance of $\mathrm{He}$ and $\mathrm{N}_{2}$ as a function of pressure differential, which is shown in Figure 2. In the presence of defects, the contribution of surface diffusion to gas transport becomes negligible, and either viscous flow or Knudsen diffusion dominates. In the case of gas transport due to viscous flow, permeance is directly proportional to the average pore pressure, which is not the case as we see that permeance of $\mathrm{He}$ and $\mathrm{N}_{2}$ is independent of pressure. Knudsen diffusion however is independent of pressure, and could be considered present if only $\mathrm{He}$ or $\mathrm{N}_{2}$ permeance is given. It is for this reason that the permeance of both $\mathrm{He}$ and $\mathrm{N}_{2}$ have been measured. In the case where Knudsen diffusion dominates, the effects of surface diffusion are negligible and we would expect to see the He permeance to be greater than that of $\mathrm{N}_{2}$. This is not the case, which shows that Knudsen diffusion is not significant, and so the membranes are considered to be defect free. The He and $\mathrm{N}_{2}$ permeance has been measured at 3 pressure differentials including the extreme pressures of interest as this number of points is sufficient to prove that the membrane is defect free, and verify the trend in permeance for both He and $\mathrm{N}_{2}$. In the case of $\mathrm{CH}_{4}$ and $\mathrm{CO}_{2}$, more points are needed to verify the trend in permeance, and so more intermediate pressure differentials were investigated.

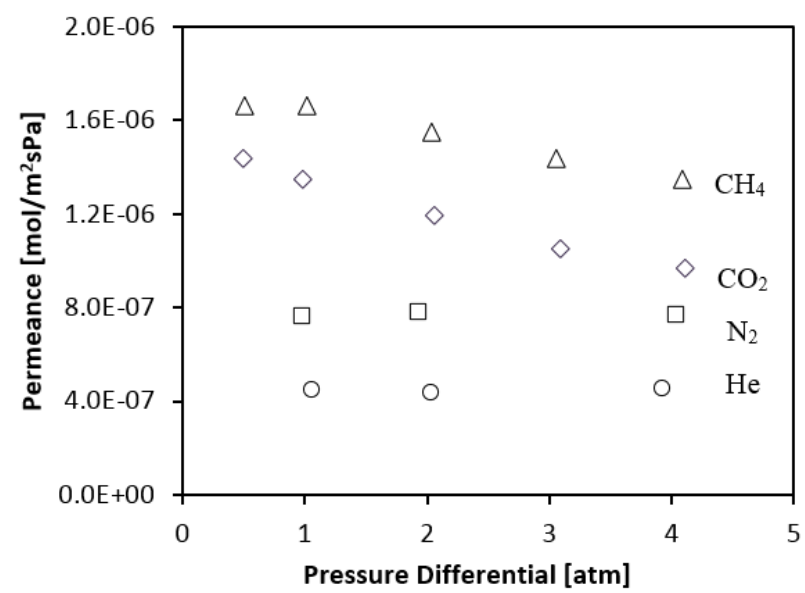

Figure 2. Single gas permeances of $\mathrm{He}, \mathrm{N}_{2}, \mathrm{CO}_{2}$, and $\mathrm{CH}_{4}$ as a function of pressure differential following exposure to each gas in turn followed by a regeneration period of 16 hours during which time the membrane is purged with He at room temperature and a pressure of 2 atm.
The decreasing permeance trends shown by $\mathrm{CO}_{2}$ and $\mathrm{CH}_{4}$ in Figure 2 can be explained in terms of the shape of their respective isotherms shown in Figure 1. More specifically, in the range of the experimental feed and permeate pressures, the $\mathrm{CO}_{2}$ and $\mathrm{CH}_{4}$ isotherms are convex (in contrast to the $\mathrm{N}_{2}$ isotherm which is approximately linear). Thus, the adsorption capacity gradient, which represents the driving force for surface diffusion, is not directly proportional to the pressure gradient. In turn, since the permeance is the pressure gradient normalized flux, in the absence of defects in the membrane structure, permeances of $\mathrm{CO}_{2}$ and $\mathrm{CH}_{4}$ are expected to decrease with increasing feed pressure. In other words, the fact that experimentally observed permeances of $\mathrm{CO}_{2}$ and $\mathrm{CH}_{4}$ decrease with feed pressure provides additional confirmation that our membranes are defect free. Using the same argument, since the $\mathrm{N}_{2}$ isotherm is approximately linear in the range of the feed and permeate pressures, its permeance was expected to be independent of the feed pressure.

The permeances shown in Figure 2 at a pressure differential of $1 \mathrm{~atm}$ for $\mathrm{He}, \mathrm{N}_{2}, \mathrm{CO}_{2}$, and $\mathrm{CH}_{4}$ are $4.38 \mathrm{x}$ $10^{-7} \pm 1.0 \times 10^{-9}, 7.71 \times 10^{-7} \pm 2.8 \times 10^{-9}, 1.35 \times 10^{-6} \pm 5.5$ x $10^{-9}$, and $1.64 \times 10^{-6} \pm 5.1 \times 10^{-9} \mathrm{~mol} / \mathrm{m}^{2} \mathrm{sPa}$ respectively. These values are greater than the single gas permeances reported in other studies for silicalite- 1 membranes at $25^{\circ} \mathrm{C}$. For $\mathrm{He}, \mathrm{N}_{2}, \mathrm{CO}_{2}$, and $\mathrm{CH}_{4}$, at $25{ }^{\circ} \mathrm{C}$, pressure unspecified, Soydaz et al. [9] found values of $0.75 \times 10^{-7}, 2.1 \times 10^{-7}, 3.5 \times 10^{-7}$, and $4.8 \times 10^{-7}$ $\mathrm{mol} / \mathrm{m}^{2} \mathrm{sPa}$ respectively. For $\mathrm{N}_{2}$ and $\mathrm{CH}_{4}$, Lovallo et al. [10] found values of $5.0 \times 10^{-8}$ and $7.0 \times 10^{-8} \mathrm{~mol} / \mathrm{m}^{2} \mathrm{sPa}$ respectively. For $\mathrm{N}_{2}$, Miachon et al. [8] found a value of 2 x $10^{-8} \mathrm{~mol} / \mathrm{m}^{2} \mathrm{sPa}$. Higher single gas permeances for $\mathrm{N}_{2}$, $\mathrm{CO}_{2}$, and $\mathrm{CH}_{4}$ were found by Algieri et al. [11], at a pressure differential of $40 \mathrm{kPa}$, and are $4.6 \times 10^{-6}, 4.3 \mathrm{x}$ $10^{-6}$, and $6.4 \times 10^{-6} \mathrm{~mol} / \mathrm{m}^{2} \mathrm{sPa}$. Wirawan et al. [12] was also able to find a greater permeance, but only for $\mathrm{CO}_{2}$ at a lower pressure differential. For $\mathrm{He}$ and $\mathrm{CO}_{2}$ at a pressure differential of $80 \mathrm{kPa}$, values of $3.06 \times 10^{-8}$, and $1.25 \times 10^{-5} \mathrm{~mol} / \mathrm{m}^{2} \mathrm{sPa}$ were found.

From the permeances shown in Figure 2, the permeance ratios for $\mathrm{CH}_{4} / \mathrm{N}_{2}, \mathrm{CO}_{2} / \mathrm{N}_{2}$, and $\mathrm{CH}_{4} / \mathrm{CO}_{2}$ at a pressure differential of $1 \mathrm{~atm}$ are $2.13,1.75$, and 1.22 . These permeance ratios are within $50 \%$ of the permeance ratios found by the authors of the studies mentioned previously. The separation of $\mathrm{CH}_{4}$ and $\mathrm{N}_{2}$ is a particularly difficult separation that is relevant to a range of natural gas upgrading processes, and many research teams are investigating membranes to be used for this separation process [13], [14]. The combination 
of this membrane's comparatively high permeance ratio, and high permeance are therefore indicative that this membrane should be further investigated for this purpose.

\subsection{Effective Diffusivities for $\mathrm{N}_{2}, \mathrm{CH}_{4}$, and $\mathrm{CO}_{2}$}

The effective diffusivities have been calculated as a function of the membrane specific constants A using the trends found from the single gas permeances shown in Figure 2, and using Equation 1.

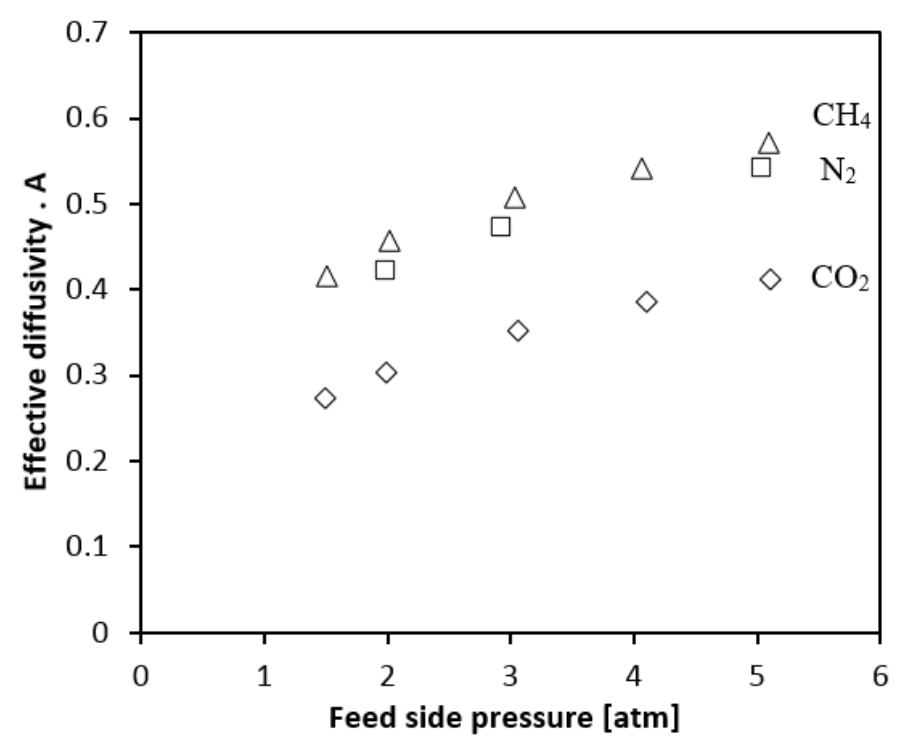

Figure 3. Effective diffusivity multiplied by constant $\mathrm{A}$ as a function of feed pressure for $\mathrm{N}_{2}, \mathrm{CO}_{2}$, and $\mathrm{CH}_{4}$

By using the adsorption capacities shown in Figure 1, Equation 1 was used to calculate the effective diffusivities of $\mathrm{CO}_{2}, \mathrm{~N}_{2}$, and $\mathrm{CH}_{4}$ as a function of the physical properties of the membrane, represented by A. The effective diffusivity ratios for the separation of $\mathrm{CH}_{4} / \mathrm{CO}_{2}, \mathrm{~N}_{2} / \mathrm{CO}_{2}$ and $\mathrm{CH}_{4} / \mathrm{N}_{2}$ are calculated, and decrease from a feed side pressure of $1 \mathrm{~atm}$ to $5 \mathrm{~atm}$ as follows, $1.54-1.38 \pm 0.007,1.44-1.32 \pm 0.007$, and $1.08-1.05 \pm 0.004$ respectively. The shape of the effective diffusivity curves agree with Figure 1 in that as the pressure increases, surface occupancy increases, and so the number of interactions between adjacent molecules increases too. Due to the increased number of interactions, it follows that diffusivity would also increase as shown in Figure 3.

Interestingly, the order of increasing effective diffusivities, $\mathrm{CO}_{2}, \mathrm{~N}_{2}$, and $\mathrm{CH}_{4}$ is not the same as the order of increasing permeance which is $\mathrm{N}_{2}, \mathrm{CO}_{2}$, and $\mathrm{CH}_{4}$. According to Equation 1, flux is directly proportional to both the effective diffusivity and the working capacity. In other words, the permeance does not solely depend on the effective diffusivity or the working capacity, but is a combination of both. It should be emphasized that unlike polymer membranes, single gas permeances of adsorbent membranes cannot be used to accurately predict the mixed gas permeation performance. With the limited number of adsorption sites, the molecules having a greater affinity towards the membrane may block access to the pores of those molecules having lower affinity towards the membrane. This blocking effect has been reported in the literature previously for binary mixtures involving $\mathrm{CO}_{2}$ [15]. To this end, mixed gas permeation experiments will be conducted for membranes of the type used in this study, and their performance will be reported in the near future.

\section{Conclusions}

Single gas permeance experiments for $\mathrm{He}, \mathrm{N}_{2}, \mathrm{CH}_{4}$, and $\mathrm{CO}_{2}$ were carried out with a defect free fabricated silicalite- 1 membrane inside a tubular $\mathrm{TiO}_{2}$ ceramic support. Their permeances were found to be $4.38 \times 10^{-7}$, $7.71 \times 10^{-7}, 1.35 \times 10^{-6}$, and $1.64 \times 10^{-6} \mathrm{~mol} / \mathrm{m}^{2} \mathrm{sPa}$, respectively at a pressure differential of $1 \mathrm{~atm}$ and a temperature of $22^{\circ} \mathrm{C}$. Given the pore size of silicalite- 1 , and the lack of membrane defects as evidenced by the greater permeance of $\mathrm{N}_{2}$ over $\mathrm{He}$, and that none of the permeances increase as a function of pressure, surface diffusion is considered to be the mechanism for gas transport through this silicalite-1 membrane. Effective diffusivities were also calculated for the membrane based on the adsorption capacity of each gas on silicalite-1, and the experimentally determined single gas permeances. For the separation of $\mathrm{CH}_{4} / \mathrm{N}_{2}, \mathrm{CO}_{2} / \mathrm{N}_{2}$, and $\mathrm{CH}_{4} / \mathrm{CO}_{2}$ the permeance ratios were found to be $2.13,1.75$, and 1.22 respectively. In order to better understand this behaviour and characterize membranes accordingly, future experiments will be conducted using binary gas mixtures.

\section{Acknowledgements}

The authors would like to acknowledge the financial support received from NSERC (Natural Sciences and Engineering Research Council) and NRCan (Natural Resources Canada). 


\section{References}

[1] J.T. Houghton, G.J. Jenkins, J.J. Ephraums, "Climate change: the IPCC scientific assessment," pp. 365, 1990.

[2] S.M. Auerbach, K.A. Carrado, P.K. Dutta, Handbook of zeolite science and technology, CRC, 2003.

[3] L.M. Robeson, "The upper bound revisited," Journal of Membrane Science, vol. 320, no. 1, pp. 390-400, 2008.

[4] P.H. Nelson, S.M. Auerbach, "Self-diffusion in singlefile zeolite membranes is Fickian at long times," Journal of Chemical Physics, vol. 110, no. 18, pp. 9235-9243, 1999.

[5] P. Li, F.H. Tezel, "Pure and binary adsorption equilibria of methane and carbon dioxide on silicalite," Separation Science and Technology, vol. 42, no. 14, pp. 3131-3153, 2007.

[6] P. Li, F.H. Tezel, "Pure and binary adsorption equilibria of carbon dioxide and nitrogen on silicalite," Journal of Chemical \& Engineering Data, vol. 53, no. 11, pp. 2479-2487, 2008.

[7] A. Ahmadpour, K. Wang, D.D. Do, "Comparison of models on the prediction of binary equilibrium data of activated carbons," AIChE Journal, vol. 44, no. 3, pp. 740-752, 1998.

[8] S. Miachon, E. Landrivon, M. Aouine, Y. Sun, I. Kumakiri, Y. Li, J. Dalmon, "Nanocomposite MFIalumina membranes via pore-plugging synthesis preparation and morphological characterisation," Journal of Membrane Science, vol. 281, no. 1-2, pp. 228-238, 2006.

[9] B. Soydaş, A. Çulfaz, H. Kalipçilar, "Characterization of zeolite membranes by gas permeation," Desalination, vol. 199, no. 1-3, pp. 371-372, 2006.

[10] M.C. Lovallo, A. Gouzinis, M. Tsapatsis, "Synthesis and characterization of oriented MFI membranes prepared by secondary growth," AIChE Journal, vol. 44, no. 8, pp. 1903-1913, 1998.

[11] C. Algieri, P. Bernardo, G. Golemme, G. Barbieri, E. Drioli, "Permeation properties of a thin silicalite-1 (MFI) membrane," Journal of Membrane Science, vol. 222, no. 1-2, 1 pp. 181-190, 2003.

[12] S.K. Wirawan, D. Creasera, J. Lindmark, J. Hedlund, I.M. Bendiyasa, W.B. Sediawan, " $\mathrm{H}_{2} / \mathrm{CO}_{2}$ permeation through a silicalite-1 composite membrane," Journal of Membrane Science, vol. 375, pp. 313-322, 2011.

[13] X. Ning, W.J. Koros, “Carbon molecular sieve membranes derived from Matrimid polyimide for nitrogen/methane separation," Carbon, vol. 66, pp. 511-522, 2014.

[14] J. Yang, J. Li, W. Wang, L. Li, J. Li, "Adsorption of $\mathrm{CO}_{2}$, $\mathrm{CH}_{4}$, and $\mathrm{N}_{2}$ on 8-, 10-, and 12-Membered Ring Hydrophobic Microporous High-Silica Zeolites: DDR, Silicalite-1, and Beta," Industrial \& Engineering Chemistry Research, vol. 52, pp. 17856-17864, 2013.

[15] L. Sandström, E. Sjöberg, J. Hedlund, "Very high flux MFI membrane for $\mathrm{CO}_{2}$ separation," Journal of Membrane Science, vol. 380, no. 1-2, pp. 232-240, 2011. 